\title{
Further experience with discriminant functions in differential diagnosis of hypercalcaemia
}

\author{
PATRICIA Fraser \\ M.D. \\ Michael Healy \\ B.A. \\ LYAL WATSON \\ F.R.C.P., F.R.A.C.P. \\ Clinical Research Centre, Division of Computing and Statistics, Northwick Park Hospital, \\ Watford Road, Harrow, Middlesex, and University College Hospital Medical School, London W.C.1
}

\begin{abstract}
Summary
Further experience has been obtained in the use of discriminant functions in the differential diagnosis of hypercalcaemia from the study of 140 new patients. The discriminant functions described in an earlier paper were derived from the results for plasma inorganic phosphate, alkaline phosphatase, chloride, bicarbonate and urea obtained from hypercalcaemic patients on admission to hospital. They indicated the correct diagnosis in ninety-two of 103 new hypercalcaemic patients $(89.3 \%)$ admitted to University College Hospital and in thirty-one of thirty-seven hypercalcaemic patients at two other hospitals.

Revised discriminant functions have been calculated including the erythrocyte sedimentation rate as a sixth variable. These revised functions have led to some improvement in diagnostic accuracy. The correct diagnosis was indicated in ninety-three of ninety-eight patients $(94.9 \%)$ at University College Hospital and in thirty-three of the thirty-seven patients at the two other hospitals.
\end{abstract}

\section{Introduction}

In an earlier study (Fraser et al., 1971) two discriminant functions derived from the results for plasma inorganic phosphate, alkaline phosphatase, chloride, bicarbonate and urea, obtained from hypercalcaemic patients on admission to hospital, led to diagnoses which coincided in 197 of 218 cases $(90.4 \%)$ with the diagnoses reached after detailed clinical investigations. It was considered that the discrimination between the various causes of hypercalcaemia might be improved by including in the functions the results of other simple tests, in particular the erythrocyte sedimentation rate (ESR), which had not been considered originally because values were not available for every patient. This additional information has been collected for new patients. The present paper describes, firstly, experience with the original discriminant functions in 140 new patients,

Requests for reprints: Dr L. Watson, University College Hospital Medical School, University Street, London W.C.1. and secondly, the results when the functions werec recalculated including the ESR.

\section{Material and methods}

Using the formulae derived in the previous study, values for the discriminant functions were calculated if from the first set of biochemical results obtained

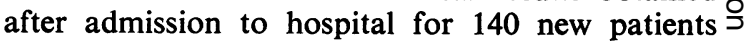
presenting with hypercalcaemia. These comprised $\vec{z}$ 103 consecutive patients investigated and treated at University College Hospital (U.C.H.) in the Metabolic Unit, thirty-two patients at Northwick Park $\vec{\theta}$ Hospital (N.P.H.) and five at the Royal Free Hosper tal (R.F.H.). In 137 cases the diagnosis was cocis firmed eventually by biopsy, at operation or pos mortem. In three cases the diagnosis was inferred from the results of radiological investigations and special tests. In the previous study 155 retrospec- $\mathbb{Q}$ tive cases were used to derive the discriminant functions and fifty cases were studied prospectively to $\frac{9}{3}$ test them. The ESR was available for 110 of these patients. In the present study the ESR was obtained for ninety-eight of the 103 new patients admitted to U.C.H. Therefore, results including the ESR were 3 . available for 208 hypercalcaemic patients investigated and treated at U.C.H. However, eighteen cases 3 . were not considered suitable for inclusion when $\delta$ recalculating the discriminant functions. There were three patients with more than one cause for their 윽 hypercalcaemia; nine cases diagnosed incorrectly by the original functions, and six cases with very extreme values for one or more variables which made them unrepresentative of their respective disease o groups. The distribution of the remaining $190 \mathrm{~N}$ patients between the same five groups as used pre- N viously is shown in Table 1 .

Discriminant analysis was carried out on these data, first using only the five biochemical variables, $\frac{\mathscr{C}}{\Phi}$ and then again including the ESR to see whether $\stackrel{\oplus}{?}$ inclusion of the additional variable in the two func- $T$ tions could improve the discrimination between the five groups of patients. 
TABLE 1. Distribution of 190 patients between five disease groups

\section{Group}

No.

(A) Hyperparathyroidism with normal alkaline phosphatase

(B) Hyperparathyroidism with raised alkaline phosphatase

(C) Non-parathyroid disease with moderate increase in urea and phosphate

(D) Non-parathyroid disease with striking increase in urea and phosphate

(E) Hyperparathyroidism with striking increase in alkaline phosphatase, urea and phosphate

Total

137

\section{Results}

In Fig. 1 the values of the two original discriminant functions have provided the co-ordinates which enable each new patient to be plotted on the graph derived in the previous study. The axes are graduated in standard deviation units and circles of radius 2.45 standard deviation units have been drawn around each group mean to represent in two dimensions the area in which $95 \%$ of the patients within each group might be expected to fall. Of the
103 new patients from U.C.H., eighty-three were placed correctly and unequivocally within a nonoverlapping part of a circle. Seven other patients were placed likewise within circles but were unequivocally incorrect. It is not surprising that a small number of patients lie outside the conventional $95 \%$ limits. If, as a simple rule, such patients are assumed to belong to the nearest disease group, five will be correctly allocated and two will be wrong. Six patients were placed where circles overlap but in only two, in the overlap between circles A and C, does the origin of their hypercalcaemia remain in doubt.

These results for the 103 patients from U.C.H. are summarized in Table 2 . In ninety-two cases $(89.3 \%)$ the statistical diagnosis based on the initial biochemical results led to correct identification of the cause of the hypercalcaemia. The statistical diagnosis was incorrect in nine cases and unhelpful in two patients placed where circles $\mathrm{A}$ and $\mathrm{C}$ overlapped. Also shown in Fig. 1 and summarized in Table 2 are the results obtained when the values of the original discriminant functions were calculated for patients at the two other hospitals.

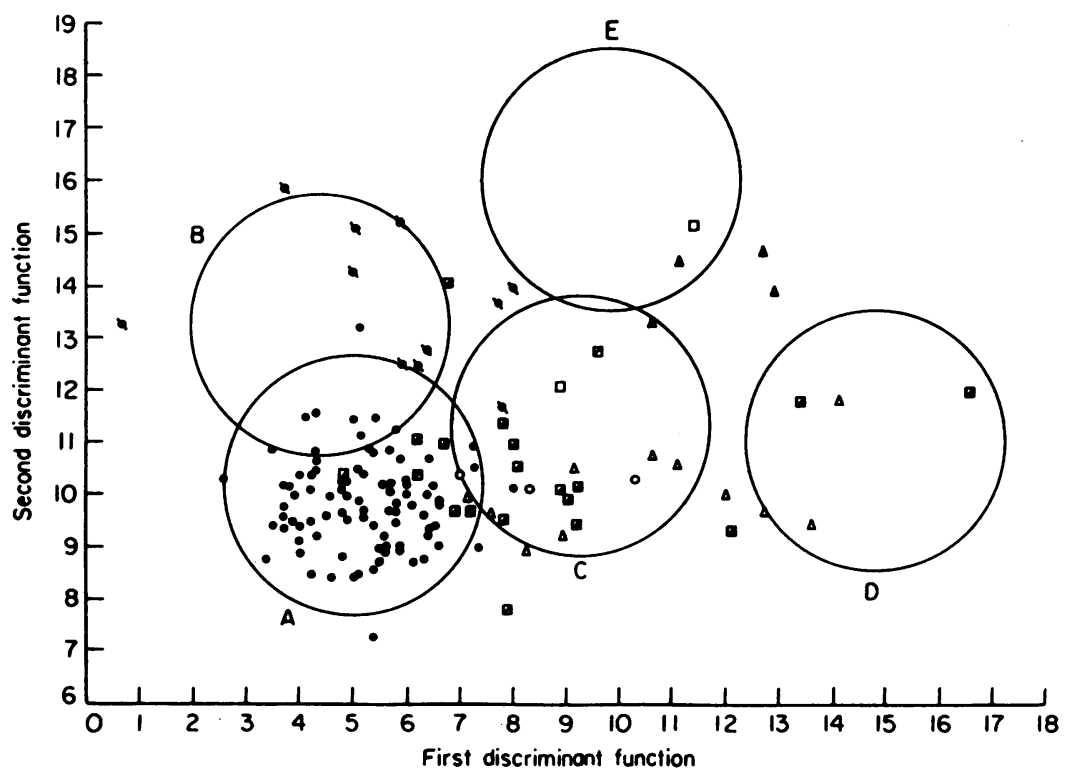

FIG. 1. Use of the original discriminant functions in 140 new patients. (The axes are graduated in standard deviation units and the circles cover $95 \%$ of the groups: (A) hyperparathyroidism with normal alkaline phosphatase; (B) hyperparathyroidism with raised alkaline phosphatase; (C) non-parathyroid disease with moderate increase in urea and phosphate; (D) non-parathyroid disease with striking increase in urea and phosphate; (E) hyperparathyroidism with striking increase in alkaline phosphatase, urea and phosphate.) (, Parathyroid adenoma or hyperplasia; $Q$, parathyroid adenoma + bone disease; $\boldsymbol{\Lambda}$, parathyroid adenoma + bone disease + renal failure; $\square$, parathyroid carcinoma; $\square$, non-parathyroid carcimona; $\square$, non-parathyroid carcinoma + bone metastases; $\triangle$, myelomatosis; $\mathbf{O}$, milk-alkali syndrome or vitamin $\mathrm{D}$ intoxication. 
TABLE 2. Comparison of the results obtained using the original and revised discriminant functions (DF)

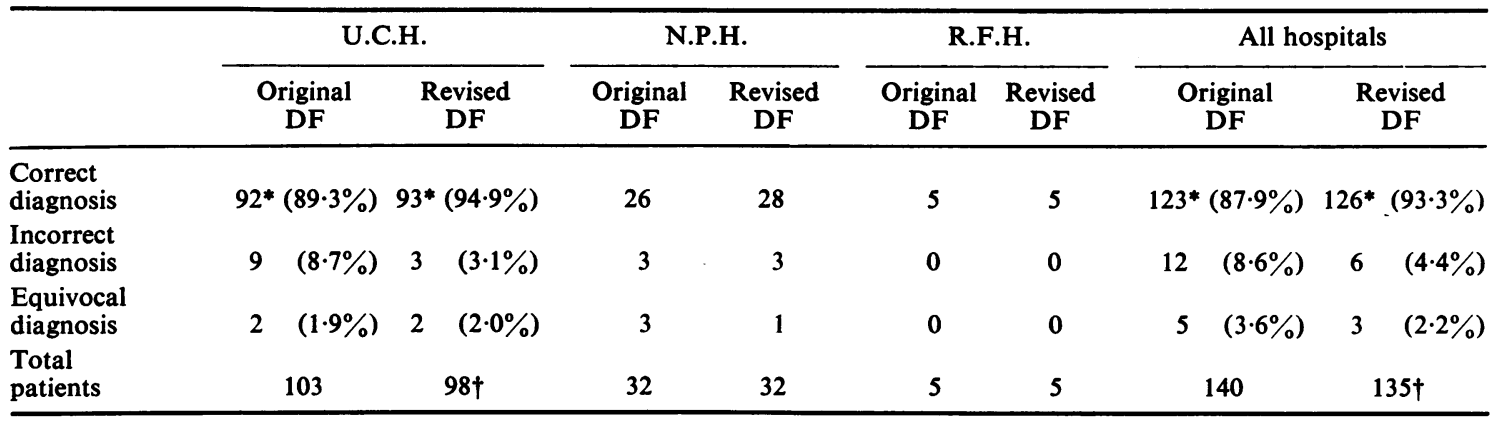

* One patient had a parathyroid adenoma and a non-parathyroid carcinoma.

+ ESR unavailable for five U.C.H. patients.

(o)

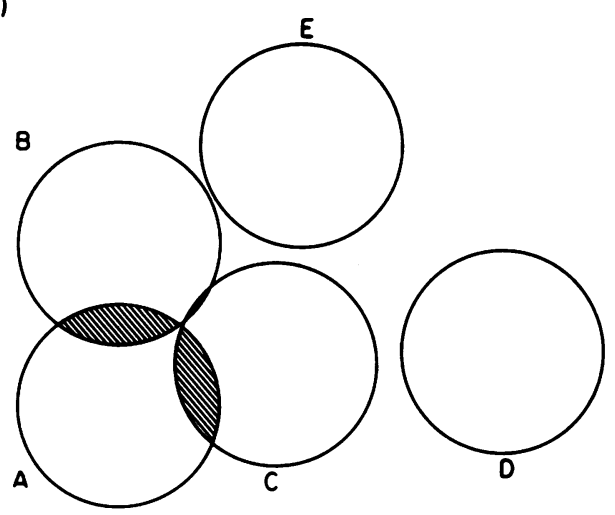

(b)

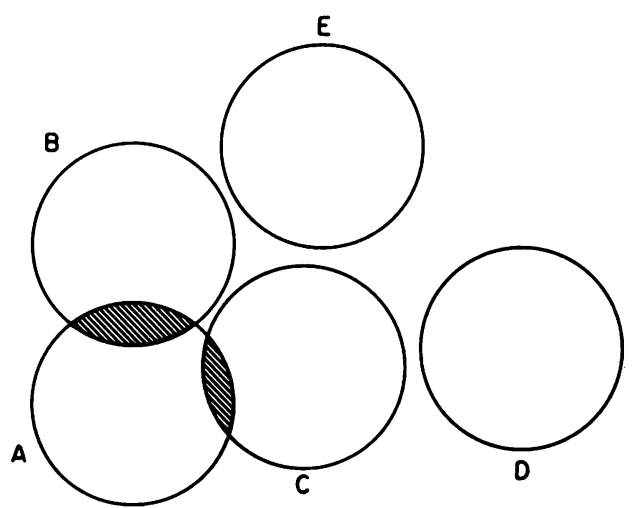

FIG. 2. (a) Relative positions of disease groups when discriminant functions contain five variables ((A)-(E) as for Fig. 1).

(b) Relative positions of disease groups when discriminant functions contain six variables $((A)-(E)$ as for Fig. 1).

In Fig. 2 the upper circles are based on the recalculated discriminant functions derived from only the five biochemical variables and the lower circles on the recalculated functions including the ESR.
The same 190 patients were included in both analyses and again the circles themselves cover areas in which $95 \%$ of the patients within each of the five groups might be expected to fall. In Fig. 2 it can be seen from the relative positions of the circles that the inclusion of the ESR has reduced the amount of $O$ overlap between the groups so that the areas of uncertainty are smaller. The recalculated discriminant functions are now weighted linear combinations of six variables and take the following form:

\section{First discriminant function}

$(1.2395 \times$ inorganic phosphate in $\mathrm{mg} / 100 \mathrm{ml})$ $\left(0.8230 \times \log _{10}\right.$ alkaline phosphatase in K.A. units. $100 \mathrm{ml})-(0.1095 \times$ chloride in $\mathrm{mEq} / \mathrm{l})+(0.0048 \times$ bicarbonate in $\mathrm{mEq} / \mathrm{l})+\left(4.2989 \times \log _{10}\right.$ urea in $\mathrm{mg} / 100 \mathrm{ml})+(0.0125 \times \mathrm{ESR}$ in $\mathrm{mm} / \mathrm{hr})$.

\section{Second discriminant function}

$-(0 \cdot 2971 \times$ inorganic phosphate in $\mathrm{mg} / 100 \mathrm{ml})-$ (3.6206 $\times \log _{10}$ alkaline phosphatase in K.A. units/ $100 \mathrm{ml})-(0 \cdot 1030 \times$ chloride in $\mathrm{mEq} / \mathrm{l})+(0 \cdot 1475 \times$ bicarbonate in $\mathrm{mEq} / \mathrm{l})-\left(1.7608 \times \log _{10}\right.$ urea in $\mathrm{mg} /$ $100 \mathrm{ml})+(0.0025 \times$ ESR in $\mathrm{mm} / \mathrm{hr})$.

In Fig. 3 the values of the revised functions have provided the co-ordinates which enable the relative positions of 135 of the 140 patients to be studied. Five patients from U.C.H. could not be placed because an ESR was unavailable, including one patient wrongly diagnosed by the previous model. $\frac{T}{0}$ The results illustrated in Fig. 3 are summarized in Table 2 and are an improvement over the results $N$ obtained with the original functions. Now, 126 of the $N$ 135 cases are correctly diagnosed $(93.3 \%)$, six are placed in the wrong disease group and three remain equivocal being in the overlap between circles $\mathrm{A}$ and C.

\section{Discussion}

The original discriminant functions calculated from the first set of biochemical results obtained 


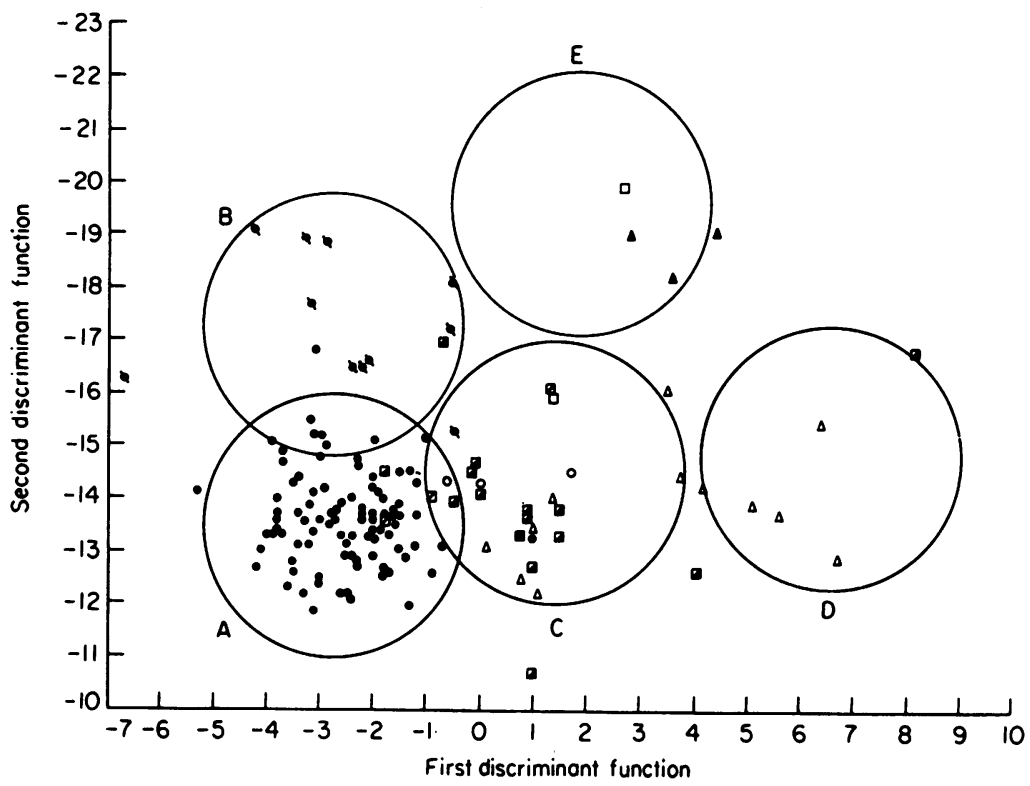

FIG. 3. Use of the revised discriminant functions in 135 new patients ((A)-(E) and legend as for Fig. 1).

after admission to hospital indicated the correct diagnosis in 123 of 140 new patients $(87.9 \%)$. In five cases the statistical analysis was unhelpful and in twelve cases $(8.6 \%)$ it was wrong. The functions were derived from data collected at U.C.H. and yet gave good results when used to analyse data collected at two other hospitals. This suggests that the technique is robust, and that the discriminant functions may be generally useful, provided that the laboratory analyses produce results which are reasonably comparable with those obtained at U.C.H.

Examination of the twelve cases in Fig. 1, in which the statistical diagnosis disagreed with the final clinical diagnosis, showed that most of the discrepancies occurred between circles A or B and C. Seven patients with malignant disease, who should have fallen in circle $C$, were placed in circle $A$, and it is possible, and was proved in one case, that the nonparathyroid tumours in some of these patients were producing a parathyroid hormone-like substance. Conversely, one patient with an adenoma and two with parathyroid disease and osteitis fibrosa were wrongly placed in circle $\mathrm{C}$, principally because of a moderate degree of renal failure. The inclusion of the ESR in the discriminant functions moved most of the non-parathyroid neoplasms previously misplaced in circle $A$ to the right, so that fewer cases were confused with the parathyroid tumours when the revised functions were used (Fig. 3).
Since the revised functions were derived from data collected at U.C.H., which included the ninety-eight new patients, one would expect them to perform well with these cases. However, the cases from the other two hospitals were not included in the derivation of these functions and therefore act as test data. The diagnostic accuracy of these cases also rose with the revised functions, from $83.7 \%$ (thirty-one out of thirty-seven) to $89.2 \%$ (thirty-three out of thirtyseven).

It is concluded that the original discriminant functions derived from the five biochemical variables continue to work well both at U.C.H. and at the two other hospitals. However, the inclusion of the ESR in the functions results in some improvement in diagnostic accuracy and therefore the revised functions are preferable.

\section{Acknowledgments}

We thank Professor C. E. Dent and Mr D. R. Davies at University College Hospital and Dr R. W. E. Watts and Dr R. Himsworth at Northwick Park Hospital for allowing us to investigate patients under their care; and Dr M. Wills for providing data from patients at the Royal Free Hospital.

\section{References}

Fraser, P., Healy, M., Rose, N. \& Watson, L. (1971) Discriminant functions in differential diagnosis of hypercalcaemia. Lancet, i, 1314. 